\title{
Simple model of shock-wave attenuation in snow
}

\author{
JEROME B. JOHNSON \\ U.S. Army Cold Regions Research and Engineering Laboratory, Fort Wainwright, Alaska 99703-7860, U.S.A.
}

\begin{abstract}
A simple momentum model, assuming that snow compacts along a prescribed pressure-density curve, is used to calculate the pressure attenuation of shock waves in snow. Four shock-loading situations are examined: instantaneously applied pressure impulses for one-dimensional, cylindrical and spherical shock-wave geometries, and a one-dimensional pressure impulse of finite duration. Calculations show that for an instantaneously applied impulse the pressure attenuation for onedimensional, cylindrical and spherical shock waves is determined by the pressure density $(\mathrm{P}-\rho)$ compaction curve of snow. The maximum attenuation for a onedimensional shock wave is proportional to $\left(X_{\mathrm{f}}-X_{0}\right)^{-1.5}$ for the multi-stage $(\mathrm{P}-$ $\rho)$ curve and $\left(X_{\mathrm{f}}-X_{0}\right)^{-2}$ when compaction occurs in a single step (single-stage compaction), where $\left(X_{\mathrm{f}}-X_{0}\right)$ is the shock-wave propagation distance. Cylindrical waves have a maximum attentuation that varies from $\left(R-R_{0}\right)^{-2}$ for single-stage compaction and $\left(R-R_{0}\right)^{-1.5}$ for multi-stage compaction, when $\left(R-R_{0}\right) \ll R_{0}$, where $R$ is the propagation radius and $R_{0}$ is the interior radius over which a pressure impulse is applied, to $R^{-4}$ when $\left(R-R_{0}\right) \gg R_{0}$. Spherical waves have a maximum attentuation that varies from $\left(R-R_{0}\right)^{-2}$ for single-stage compaction and $(R-$ $\left.R_{0}\right)^{-1.5}$ for multi-stage compaction to $R^{-6}$ when $\left\langle R-R_{0}\right\rangle \gg R_{0}$.

The shock-wave pressure in snow for a finite-duration pressure impulse is determined by the pressure impulse versus time profile during the time interval of the impulse. After the pressure impulse ends, shock-wave pressure attentuation is the same as for an instantaneously applied pressure impulse containing the same total momentum. Pressure attentuation near a shock-wave source, where the duration of the shock wave is relatively short, is greater than for a shock wave farther from a source where the shock wave has a relatively long duration. Shock-wave attenutation in snow can be delayed or reduced by increasing the duration of a finite-duration pressure impulse. A sufficiently long-duration impulse may result in no shock-wave pressure attenuation in a shallow snow cover.
\end{abstract}

\section{NOMENCLATURE}

$a$

$a_{i}$

squarewave segments.

$A_{i} \quad\left[V_{i-1}{ }^{2}\left(X a_{i}\right)-\gamma b_{i}\right] X_{a_{i}}{ }^{2}$

$b_{i} \quad$ Weighting coefficients determined from $f(t)$.

$B_{i} \quad \gamma b_{i}$.

$D \quad$ Shock-wave propagation speed.

$d_{\mathrm{LC}} \quad$ Distance from a line charge.

$f(t) \quad$ Function describing the shape of a variable pressure impulse.

$H_{\mathrm{p}} \quad$ Total momentum per unit area applied to the snow.

$H_{\mathrm{p} i} \quad$ Momentum per unit area applied to the snow in the $i$ th square-wave segment.

$H_{\mathrm{s}}, \mathrm{d} H_{\mathrm{s}} \quad$ Momentum per unit area in the snow and its derivative.

$I_{0}$ Instantaneously applied pressure impulse.
$M\left(X_{\mathrm{f}}\right)$

$m, \mathrm{~d} m$

$P$

$P_{0}$

$P(t)$

$r$

$R$

$R_{0}$

$t, \mathrm{~d} t$

$t_{\mathrm{f}}$

$t_{0}$

$U_{0}$

V

$V_{i}$

$X_{a}$

$X_{\mathrm{a}_{i}}$
Total mass of the compacted material. Mass per unit area and its derivative. Shock-wave pressure.

Maximum pressure amplitude of the shock wave.

Shock-wave pressure amplitude as a function of time.

Current position of the inner radius of a cavity surface.

Shock-wave propagation radius.

Initial radius of a cavity surface in the snow.

Time and its derivative.

Time required for the shock wave to reach $X_{\mathrm{f}}$.

Time at which the pressure impulse is applied.

Distance snow surface has moved.

Snow-particle velocity.

Snow-particle velocity in the $i$ th square-wave segment.

Shock-wave front position at $t=a$.

Shock-wave front position at $t=a_{i}$. 
$X_{\mathrm{f}}$

Shock-wave front position; $X_{\mathrm{f}}=X_{\mathrm{f}}-X_{0}$ for $X_{0}=0$.

$X_{\mathrm{i}}$

$X_{0}$

$\alpha$

$\beta$

$\gamma$

$\epsilon$

$\rho_{\mathrm{f}}$

$\rho_{0}$

Current snow-surface position.

Initial snow-surface position.

Decay coefficient.

Pressure-dependent relative snow compaction $\left(1-\rho_{0} / \rho_{\mathrm{f}}\right)$.

$P_{0} \beta / \rho_{\mathrm{f}}(1-\beta)$.

$R-R_{0}$. Initial snow density.
Pressure-dependent compacted snow density.

\section{INTRODUCTION}

An understanding of shock-wave attenuation (that is, the pressure-amplitude reduction of a shock wave over a given propagation distance) in snow is needed to solve a number of problems in planetary science, cold regions and military engineering, shock-wave isolation, and seismic monitoring. For example, astronomers are interested in determining the stresses associated with impacts between low-density frost clouds and planetary bodies, avalanche-control professionals are interested in knowing the effectiveness of explosives in initiating avalanches, and military engineers are primarily interested in the extent to which their explosive methods are degraded in snow-covered terrain. Shock-wave isolation, using snow, is a technique for protecting an object from shock damage by causing shock waves to attenuate in a protective layer of snow around the object.

The mechanical properties of snow for high strainrate, large-amplitude shocks are not now well understood. Consequently, attempts to estimate shock-wave attenuation in snow have relied on field measurements of pressure attentuation from explosions in snow and on theoretical constitutive descriptions. Mellor (1977) used the results from field measurements of explosive detonations in snow to estimate the attenuating properties of snow for spherical shock waves. He estimated that the attenuation of shock-wave pressure, combining geometric spreading and internal dissipation, near an explosion attenuates as $R^{-4}$ ( $R$ being the propagation radius), and close to the elastic limit the attenuation is approximately an $R^{-3}$ attenuation. Brown $(1980,1981,1983)$ estimated shock-wave attenuation for plane waves in snow. He used two theoretical volumetric constitutive laws, one for medium- to high-density snow and one for low-density snow, to estimate shock-wave attenuation. His results indicate that plane shock waves can attenuate by more than $80-90 \%$ after propagating through only $0.06-0.1 \mathrm{~m}$ of snow. These are large attenuations that are, at their maximum, proportional to $\left(X_{\mathrm{f}}-X_{0}\right)^{-1.2}\left(\left[X_{\mathrm{f}}-X_{0}\right]\right.$ is the propagation distance).

Some of the difficulties in determining shock-wave attenuation in snow are that the attenuation is affected by the pressure, geometry and duration of a shock wave, in addition to the mechanical properties of the snow. The objective of this paper is to use a simple analytical model to show the influence of a pressure impulse (the integral of pressure over the time interval during which the pressure acts) and its geometry on shock-wave pressure attenuation, in addition to the effects caused by the mechanical behavior of snow. The effects of shock-wave geo- metry are accounted for by calculating the attenuation of plane, cylindrical and spherical shock waves in snow. The effects of shock-wave duration and initial pressure amplitude are analyzed by applying a variable pressuretime profile shock impulse to the snow and calculating pressure as a function of shock-wave propagation distance.

\section{MOMENTUM MODEL}

Shock-wave attenuation, in this model, is the result of a mechanical process in which attenuation occurs through momentum transfer from an applied pressure impulse to the snow. The momentum model, also known as the "snow-plow" model, was used in early attempts to develop constitutive relations to analyze the dynamic behavior of porous materials (Herrmann, 1971). The porous material is assumed to compact to its final density at a negligible stress and then be incompressible (an ideal single-stage locking material). The snow-plow model can be extended to include porous materials that compact along a prescribed pressure-density $(\mathrm{P}-\rho)$ curve. Pressure changes in the porous material will cause it to collapse to a density consistent with the $(\mathrm{P}-\rho)$ curve. After compaction, the material is assumed to be incompressible until pressure changes cause a collapse to a new density state (an ideal multi-stage locking material (Hanagud, 1967)). The change in momentum caused by a pressure impulse is spread uniformly over the mass of the compressed material producing a constant pressure and particle velocity in the mass behind an advancing shock wave (Herrmann, 1971). The shock wave is lengthened in time and reduced in amplitude as more of the material is compacted by the propagating shock. Hence, attenuation is caused by momentum spreading. Attenuation attributable to plastic deformation, fracturing and release waves are not considered. Although plastic deformation and fracturing may significantly affect the behavior of snow, the compaction is treated phenomenologically since little data exist from which to determine the mechanisms that produce a given compaction curve. Release waves (tensile waves generated at boundaries or following a pressure impulse) are also an important source of attenuation. The momentum model, presented here, is not capable of treating attenuation caused by release waves. A following paper using experimentally derived compaction data for snow will treat the more complicated problem of release-wave attenuation. In this paper, attenuation solutions are first developed for a single-stage locking material and then extended to include a multi-stage locking material.

\section{INSTANTANEOUSLY APPLIED IMPULSE}

Consider a pressure impulse $\left(I_{0}\right)$ applied normal to the plane surface of snow, and assume that snow is an ideal single-stage locking material. Snow next to the plane surface will be immediately compacted to its defined final density stage. Since the compacted snow is rigid, it will move at a uniform pressure and particle velocity after the pressure impulse is applied. The stress wave will propagate into the snow as a compaction shock wave, moving with a velocity $D$ at a pressure $P$, and particle velocity $V$. At the shock front, these parameters are 


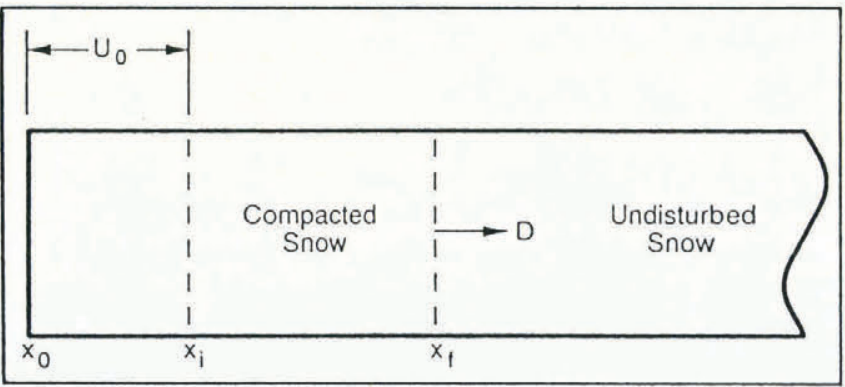

Fig. 1. Deformation geometry for plane shockwave propagation in snow.

related by the Rankine-Hugoniot jump conditions for the conservation of mass and momentum across the shock front (Kolsky, 1963)

$$
\rho_{0} D=\rho_{\mathrm{f}}[D-V]
$$

and

$$
P=\rho_{0} D V
$$

where $\rho_{0}$ is the initial density of the snow and $\rho_{\mathrm{f}}$ is the compacted snow density.

Figure 1 shows the deformation geometry in one dimension for a pressure impulse applied at the initial snow surface $X_{0}$. The location of the surface of the snow and the shock front at some time after the application of the pressure impulse are $X_{\mathrm{i}}$ and $X_{\mathrm{f}}$, respectively. During the time that the shock front has traveled to $X_{\mathrm{f}}$, the snow surface has moved a distance $U_{0} . U_{0}$ is a function of time or, alternatively, a function of shock-wave propagation position $X_{\mathrm{f}}$. At any time, the location of the snow surface is

$$
X_{\mathrm{i}}=X_{0}+U_{0} .
$$

The displacement of the snow surface can be calculated by integrating the particle velocity over time using

$$
U_{0}=\int_{t_{0}}^{t_{f}} V \mathrm{~d} t
$$

where $t_{0}$ is the time that the pressure impulse is applied and $t_{\mathrm{f}}$ denotes the time required for the shock wave to reach $X_{\mathrm{f}}$. The equation describing shock-wave attentuation can be formulated as a function of time or shock-wave propagation distance. Knowing the shockwave pressure attentuation as a function of propagation distance is of more practical interest than following the pressure as a function of time. Shock-wave attenuation as a function of propagation distance can be developed by reformulating the snow-surface displacement in terms of shock-wave position rather than time by using

$$
D=\frac{\mathrm{d} x}{\mathrm{~d} t}
$$

and rewriting Equation (4) as

$$
U_{0}=\int_{X_{0}}^{X_{\mathrm{f}}} \frac{V}{D} \mathrm{~d} x .
$$

From Equation (1)

$$
\frac{V}{D}=\left(1-\frac{\rho_{0}}{\rho_{\mathrm{f}}}\right)=\beta
$$

where $\beta$ describes the relative snow compaction (the mechanical behavior of the snow). Substituting Equation (7) into Equation (6) and solving for the position of the surface at time $t_{\mathrm{f}}$ as a function of $X_{0}$ and $X_{\mathrm{f}}$ gives

$$
X_{\mathrm{i}}=(1-\beta) X_{0}+\beta X_{\mathrm{f}} .
$$

The mass and the momentum per unit area of the snow contained in a length $\mathrm{d} x$ between the current snowsurface position $X_{\mathrm{i}}$ and shock-front position $X_{\mathrm{f}}$ are

$$
\mathrm{d} m=\rho_{\mathrm{f}} \mathrm{d} x
$$

and

$$
\mathrm{d} H_{\mathrm{s}}=\rho_{\mathrm{f}} V \mathrm{~d} x .
$$

Since the compacted snow is assumed to be rigid, $V$ and $\rho_{\mathrm{f}}$ are constant through the region $X_{\mathrm{i}}$ to $X_{\mathrm{f}}$, where $V$ is equal to the particle velocity at $X_{\mathrm{f}}$, i.e. $V=V\left(X_{\mathrm{f}}\right)$. Consequently, the momentum per unit area in the snow at the time the shock wave has reached $X_{\mathrm{f}}$ is

$$
H_{\mathrm{s}}=\int_{X_{\mathrm{i}}}^{X_{\mathrm{f}}} \rho_{\mathrm{f}} V \mathrm{~d} x=V \rho_{\mathrm{f}}\left(X_{\mathrm{f}}-X_{\mathrm{i}}\right) .
$$

Substituting Equation (8) into Equation (11) gives

$$
H_{\mathrm{s}}=\rho_{\mathrm{f}}(1-\beta) V\left(X_{\mathrm{f}}-X_{0}\right) \text {. }
$$

The momentum per unit area in the snow $H_{\mathrm{s}}$ must be equal to the momentum per unit area applied to the snow from the instantaneously applied pressure impulse that is given by

$$
H_{\mathrm{p}}=I_{0} \text {. }
$$

Equating $H_{\mathrm{s}}$ and $H_{\mathrm{p}}$ and solving for $V$ gives

$$
V=\frac{I_{0}}{\rho_{0}\left(X_{\mathrm{f}}-X_{0}\right)} .
$$

Using Equations (7) and (14) in Equation (2) to determine the pressure at the shock front gives

$$
P\left(X_{\mathrm{f}}\right)=\frac{\rho_{0} V^{2}}{\beta}=\frac{I_{0}{ }^{2}}{\rho_{0} \beta\left(X_{\mathrm{f}}-X_{0}\right)^{2}} .
$$

Figure 2 shows the shock-front pressure for snow subjected to an instantaneous pressure impulse and compaction to a final density occurring in one step (single stage). The pressure attenuates as $\left(X_{\mathrm{f}}-X_{0}\right)^{-2}$, with proportionality constant determined by pressure impulse, initial snow density and relative snow compaction. The proportionality constant determines the pressure amplitude that the snow is capable of supporting at a given $\left(X_{\mathrm{f}}-X_{0}\right)$, but not the attenuation with distance. Increasing the pressure-impulse amplitude produces a greater momentum transfer to snow that results in higher shock-wave pressures at a given propagation 


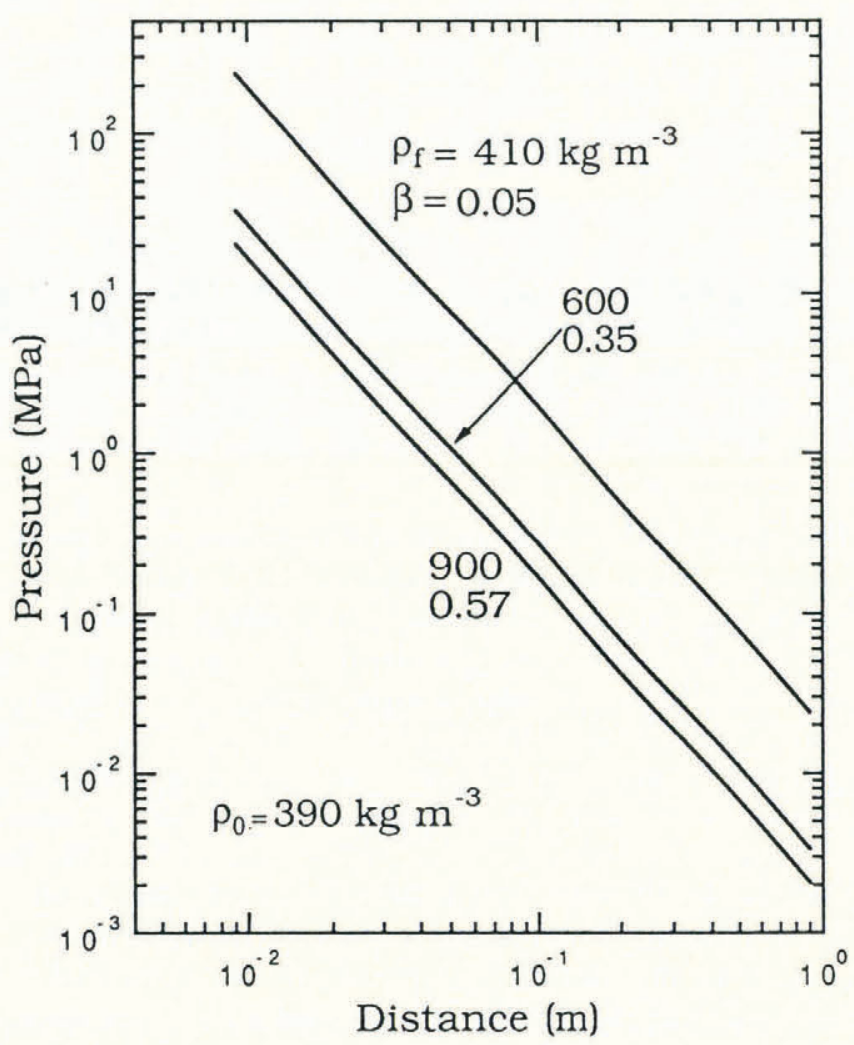

Fig. 2. Pressure attenuation with distance for an instantaneously applied plane shock-wave pressure impulse (605 Pas), predicted by the snow-plow model using single-stage snow compactions from an initial density of $390 \mathrm{~kg} \mathrm{~m}^{-3}$ to 410,600 and $900 \mathrm{~kg} \mathrm{~m}^{-3}$.

distance, $\rho_{0}$ and $\beta$ remaining constant. A larger $\beta$, with constant $I_{0}$ and $\rho_{0}$, causes a lower shock-wave propagation velocity at a given $\left(X_{\mathrm{f}}-X_{0}\right)$, as required by massconservation Equation (7), which results in reduced pressures in the snow.

When snow compaction is pressure-dependent (Fig. $3)$, the momentum equation (Equation (11)) cannot be directly integrated as $\rho_{\mathrm{f}}$ is not constant through the region $X_{\mathrm{i}}$ to $X_{\mathrm{f}}$. Since the material behind the shock wave is rigid, $V$ is constant from $X_{\mathrm{i}}$ to $X_{\mathrm{f}}$ and the momentum (Equation (11)) is

$$
H_{\mathrm{s}}=V \int_{X_{\mathrm{i}}}^{X_{\mathrm{f}}} \rho_{\mathrm{f}} \mathrm{d} x
$$

where

$$
\int_{X_{\mathrm{i}}}^{X_{\mathrm{f}}} \rho_{\mathrm{f}} \mathrm{d} x=M\left(X_{\mathrm{f}}\right)
$$

is the mass of the compacted material behind the shock wave. Although the density varies over the limits of integration, it is only the total mass behind the shock wave that is needed to determine the momentum. The mass can be determined from

$$
M\left(X_{\mathrm{f}}\right)=\rho_{\mathrm{f}}\left(X_{\mathrm{f}}\right)\left(X_{\mathrm{f}}-X_{\mathrm{i}}\right)
$$

where $\rho_{\mathrm{f}}\left(X_{\mathrm{f}}\right)$ is the final density at the shock front and $X_{\mathrm{i}}$ is determined from Equation (8). The pressure and

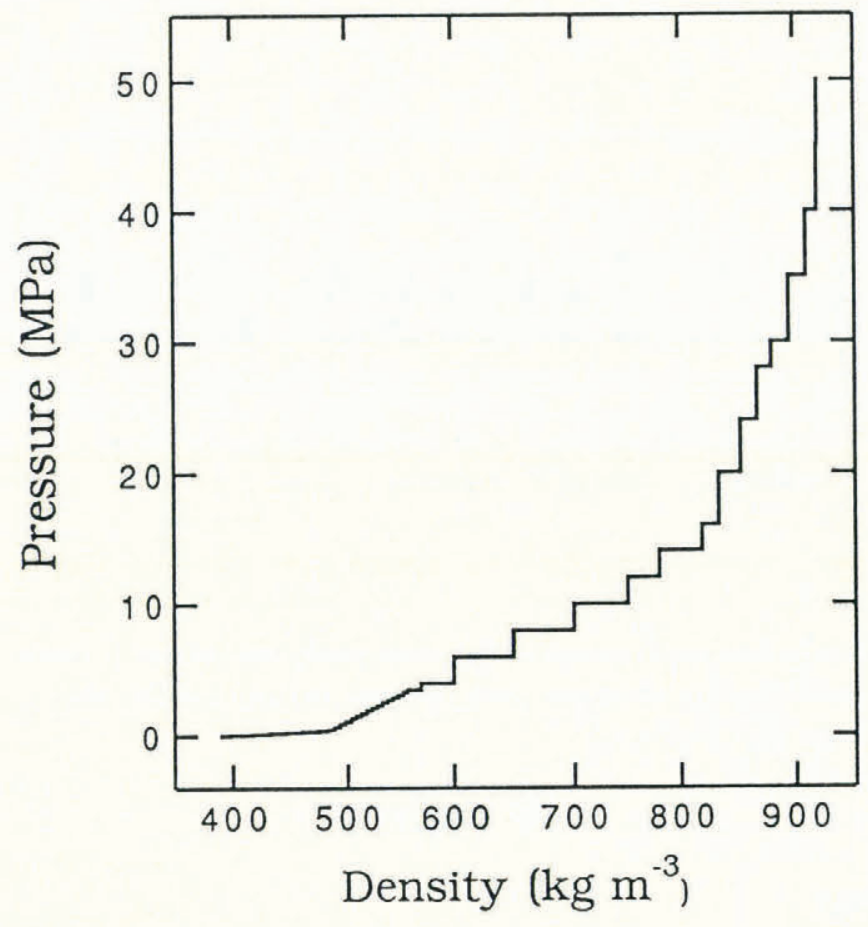

Fig. 3. Pressure-density curve (multi-stage compaction curve) for snow with an initial density of $390 \mathrm{~kg} \mathrm{~m}^{-3}$ derived from experimental data (Johnson and others, 1990).

$\rho_{\mathrm{f}}\left(X_{\mathrm{f}}\right)$ at the shock front are determined by solving Equation (15) iteratively until $P\left(X_{\mathrm{f}}\right)$ and $\rho_{\mathrm{f}}\left(X_{\mathrm{f}}\right)$ lie on the specified $(\mathrm{P}-\rho)$ compaction curve (Fig. 3). Shockpressure attentuation is approximately $\left(X_{\mathrm{f}}-X_{0}\right)^{-1.5}$ for the prescribed multi-stage compaction $(\mathrm{P}-\rho)$ curve used in this study as compared to $\left(X_{\mathrm{f}}-X_{0}\right)^{-2}$ for single-stage compaction (impulse B compared to impulse A; Fig. 4). The pressure attentuation with distance is a direct result of the form of the compaction curve and may be different for various snow types. A single-stage compaction will always have a larger attentuation with distance than a multi-stage compaction. For single-stage compaction, $\rho_{\mathrm{f}}$ will remain constant as the shock pressure decreases whereas for multi-stage compaction $\rho_{\mathrm{f}}$ decreases with a decrease in pressure.

\section{CONSTANT-PRESSURE IMPULSE OF FINITE DURATION}

The solution given by Equation (15) is not a very satisfying way of evaluating shock-wave attenuation, since it assumes instantaneous application of the pressure impulse. This is unrealistic, as it implies infinite pressure at $x=X_{0}$, and does not show how pressure impulses with different amplitudes and durations, but each having the same total momentum, are attenuated. An analytical solution can be derived for a constant-pressure impulse of finite duration, given by

$$
\begin{aligned}
P(t) & =P_{0} & & 0 \leq t \leq a \\
p(t) & =0 & & a<t
\end{aligned}
$$

where $a$ is the duration of the applied pressure pulse. 


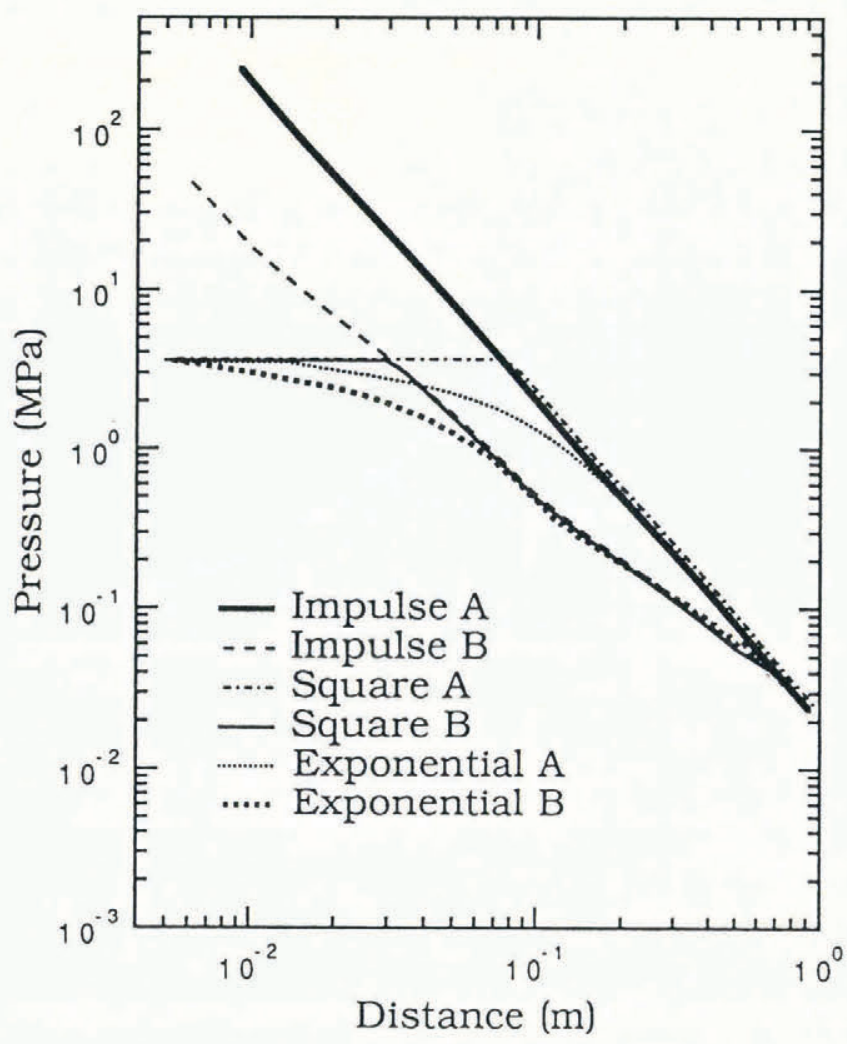

Fig. 4. Comparison of pressure attenuation with distance in snow with an initial density of $390 \mathrm{~kg} \mathrm{~m}^{-3}$ for plane shock waves, each having a total applied pressure impulse of $605 \mathrm{Pas}$, predicted by the snow-plow model. The pressure impulse was applied instantaneously for impulse $A$ (single-stage compaction) and impulse B (multi-stage compaction), with a square wave of $0.17 \mathrm{~ms}$ duration for square $A$ (single-stage compaction) and square $B$ (multi-stage compaction), and an exponential pulse of $1.6 \mathrm{~ms}$ duration for exponential A (single-stage compaction) and exponential B (multi-stage compaction).

The momentum per unit area caused by the pressure impulse is

$$
H_{\mathrm{p}}=\int_{0}^{t} P_{0} \mathrm{~d} t \quad \text { for } 0 \leq t \leq a
$$

and

$$
H_{\mathrm{p}}=\int_{0}^{a} P_{0} \mathrm{~d} t \quad \text { for } a<t .
$$

Equations (17) and (18) show that the momentum during application of the pressure impulse will vary with time, while the momentum after the impulse has been applied is constant. Therefore, separate solutions for shock-wave pressure attenuation are needed during the time period of pulse application and for the time period after the pulse has been applied. Equations (17) and (18) can be transformed into spatial coordinates by use of the results of Equations (5) and (7), giving

$$
H_{\mathrm{p}}=\int_{0}^{X_{\mathrm{f}}-X_{0}} P_{0} \frac{\beta}{V} \mathrm{~d} x \quad \text { for } 0<X_{\mathrm{f}}-X_{0} \leq \frac{a V}{\beta} .
$$

The limits of integration are 0 to $X_{\mathrm{a}}=a V / \beta$. Equating momentum in the snow (Equation (12)) to the pressureimpulse momentum (Equation (19)), letting $X_{0}=0$ and differentiating gives

$$
\frac{P_{0} \beta}{\rho_{\mathrm{f}}(1-\beta)}=V \frac{\mathrm{d}}{\mathrm{d} x}\left[V X_{\mathrm{f}}\right]=\frac{1}{X_{\mathrm{f}}} \frac{\mathrm{d}}{\mathrm{d} x}\left[\frac{1}{2} V^{2} X_{\mathrm{f}}^{2}\right] .
$$

Integrating Equation (20) gives the particle velocity

$$
V=\left[\frac{p_{0} \beta}{\rho_{\mathrm{f}}(1-\beta)}\right]^{\frac{1}{2}} \quad \text { for } 0 \leq X_{\mathrm{f}} \leq X_{a} .
$$

Hence, during the period of pressure impulse application, the density, relative snow compaction and particle velocity are constant and there is no attenuation of the shock-wave pressure. The distance that the shock wave has traveled at $t=a$ is given by

$$
X_{a}=D a=\left[\frac{P_{0} a^{2}}{\beta \rho_{\mathrm{f}}(1-\beta)}\right]^{\frac{1}{2}} .
$$

After the pressure-impulse momentum has been applied to the snow, the relationship between the pressureimpulse momentum and snow momentum is

$$
P_{0} a=\rho_{\mathrm{f}} V(1-\beta) X_{\mathrm{f}} \quad \text { for } X_{a}<X_{\mathrm{f}} \text {. }
$$

Solving for the particle velocity and using the definition of $X_{a}$ in Equation (22) gives

$$
V=\left[\frac{P_{0} a}{\rho_{\mathrm{f}}(1-\beta) X_{\mathrm{f}}}\right] \text {. }
$$

The shock-wave pressure as a function of distance can now be determined from Equations (2), (7), (21) and (24), giving

$$
\begin{array}{ll}
P\left(X_{\mathrm{f}}\right)=P_{0} & \text { for } 0 \leq X_{\mathrm{f}} \leq X_{a} \\
P\left(X_{\mathrm{f}}\right)=\frac{P_{0}{ }^{2} a^{2}}{\beta \rho_{0} X_{\mathrm{f}}{ }^{2}} & \text { for } X_{a}<X_{\mathrm{f}} .
\end{array}
$$

Since $P$ is constant while the pressure impulse is being applied, $\rho_{\mathrm{f}}$ and $\beta$ are also constant and $X_{a}$ can be directly calculated for either single-stage or multi-stage compaction. After the pressure impulse has ended, solving Equation (25b) for multi-stage compaction requires that $\rho_{\mathrm{f}}\left(X_{\mathrm{f}}\right)$ be iterated until $\rho_{\mathrm{f}}\left(X_{\mathrm{f}}\right)$ and $P\left(X_{\mathrm{f}}\right)$ lie on the $(P-$ p) curve (Fig. 3), as was the case for the instantaneously applied pressure impulse. Figure 4 shows the shockwave attenuation for single-stage compaction (square A) and multi-stage compaction (square B). The shock wave does not begin attenuating until after all of the pressureimpulse momentum has been applied to the snow surface, and then it attenuates as $X_{\mathrm{f}}^{-2}$ for the single-stage compaction and approximately as $X_{\mathrm{f}}^{-1.5}$ for the multistage compaction curve. The propagation distance at a constant shock-wave pressure differs between squares $\mathrm{A}$ and $\mathrm{B}$. This difference is controled by the amount of relative snow compaction. With a large amount of compaction, the shock wave propagates a shorter distance before it attenuates than it does for a smaller amount of 
compaction. Comparison of the square-wave pressureimpulse (squares A and B) to an instantaneous pressure impulse (impulses $\mathrm{A}$ and $\mathrm{B}$ ) shows that the pressure is lower for the square wave during its duration of application than for an instantaneously applied pressure impulse. After the square-wave momentum has been completely transferred to the snow, both the square wave and instantaneously applied impulse pressures are the same. Changing the duration of a square-wave pressure impulse with constant total momentum will also affect shock-wave pressure attenuation. Decreasing the duration of an impulse causes the initial pressure of a shock wave to increase. The shock wave will also being attenuating at $X_{\mathrm{f}}^{-2}$, for single-stage compaction, and $X_{\mathrm{f}}^{-1.5}$, for multi-stage compaction, earlier.

\section{VARIABLE PRESSURE IMPULSE OF FINITE DURATION}

Simulating a realistic applied pressure impulse requires a function that can represent variable pressure impulses of finite duration. These variable-pressure impulses must be modeled by non-linear differential equations that do not have closed-form solutions and whose numerical solutions can be unstable (Hornbeck, 1971). In addition, the form of the differential equations depends on the form of the pressure impulse applied to the snow surface. To reduce these computational difficulties, here the variable pressure impulses were approximated by a sequence of square waves. The accuracy of such an approximation depends on the duration specified for each square-wave segment comprising the total applied pressure impulse. Instead of exactly formulating the problem and obtaining a numerical solution, the pressure impulse is approximated and solved analytically within the sequential time steps. The variable-pressure impulse of finite duration beginning at $t=0$ is approximated by

$$
\begin{array}{ll}
b_{0}=1 & \text { for } 0 \leq t<a_{1} \\
b_{1}=f\left(\frac{a_{2}+a_{1}}{2}\right) & \text { for } a_{1} \leq t<a_{2}
\end{array}
$$

$$
\begin{aligned}
P(t)=p_{0} b_{i} & & \\
b_{n-1}=f\left(\frac{a_{n}+a_{n-1}}{2}\right) & & \text { for } a_{n-1} \leq t<a_{n} \\
b_{n}=0 & & \text { for } a_{n} \leq t .
\end{aligned}
$$

A smooth curve, $f(t)$, fitted to the sequence of square waves passes through a series of points, $b_{i}$ (weighting coefficients), at the midpoint between the beginning time and ending time for each square-wave segment $f\left[\left(a_{i+1}+\right.\right.$ $\left.\left.a_{\mathrm{i}}\right) / 2\right]$, where each square-wave segment ends at time $a_{i}$ where $i=1,2,3, \ldots, n-1$ (Fig. 5)).

Solving for the pressure in snow as a function of distance, using Equation (26), requires that the problem be formulated for three conditions. The first is for the time, or spatial increment, of the first square-wave segment. The next solution must account for the momentum that has been applied to the snow at the time, or spatial position, of interest. Finally, a solution must be found after all of the pressure-impulse momentum has been applied

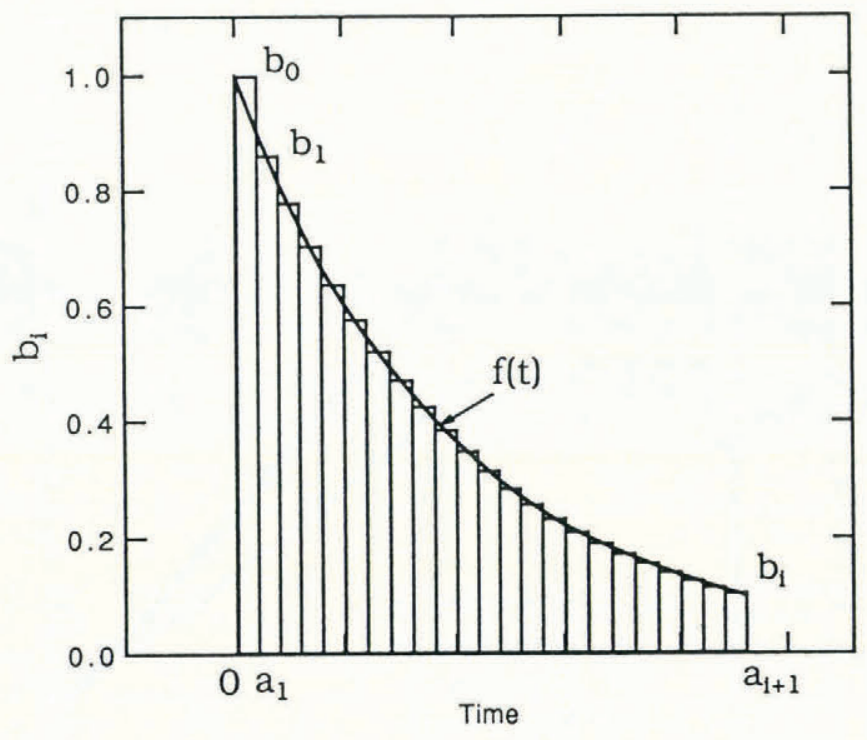

Fig. 5. Weighting coefficients and $f(t)$ as a function of time for an exponential pressure impulse.

to the snow. The conservation of momentum conditions, for $X_{0}=0$, can be expressed as

$$
\begin{aligned}
& \int_{0}^{X_{\mathrm{f}}} P_{0} b_{0} \frac{\beta}{V_{0}(x)} \mathrm{d} x=\rho_{\mathrm{f}} V_{0}\left(X_{\mathrm{f}}\right)(1-\beta) X_{\mathrm{f}} \\
& \text { for } 0 \leq X_{\mathrm{f}}<X_{a_{1}} \\
& H_{p_{0}}+\int_{X_{a_{1}}}^{X_{\mathrm{f}}} P_{0} b_{1} \frac{\beta}{V_{1}(x)} \mathrm{d} x=\rho_{\mathrm{f}} V_{1}\left(X_{\mathrm{f}}\right)(1-\beta) X_{\mathrm{f}} \\
& \text { for } X_{a_{1}} \leq X_{\mathrm{f}}<X_{a_{2}} \\
& \sum_{i=0}^{n-2} H_{p_{i}}+\int_{X_{a_{n-1}}}^{H_{\mathrm{f}}} p_{0} b_{n-1} \frac{\beta}{V_{n-1}(x)} \mathrm{d} x=\rho_{\mathrm{f}} V_{n-1}\left(X_{\mathrm{f}}\right)(1-\beta) X_{\mathrm{f}} \\
& \text { for } X_{a_{n-1}} \leq X_{\mathrm{f}}<X_{a_{n}} \\
& \sum_{i=0}^{n-1} H_{p_{i}}=\rho_{\mathrm{f}} V_{n}\left(X_{\mathrm{f}}\right)(1-\beta) X_{\mathrm{f}}
\end{aligned}
$$

for $X_{a_{n}} \leq X_{\mathrm{f}} \cdot(27 \mathrm{c})$

There are $n+1$ equations to solve before the complete particle-velocity solution can be found. Each equation depends on the solutions of the previous segments.

Equation (27a) is a constant-pressure impulse with a particle velocity of

$$
V_{0}{ }^{2}\left(X_{\mathrm{f}}\right)=\gamma \quad \text { for } 0 \leq X_{\mathrm{f}}<X_{a_{1}}
$$

where

$$
X_{a_{1}}{ }^{2}=\gamma \frac{a_{1}{ }^{2}}{\beta^{2}}
$$

and

$$
\gamma=\frac{P_{0} \beta}{\rho_{\mathrm{f}}(1-\beta)} .
$$

Equation (27b) can be solved sequentially to give a set of recursion relations for position, and particle velocity 


$$
\begin{aligned}
& X_{a_{i+1}}=\left[a_{i+1}-a_{i}+\frac{\beta}{B_{i}}\left(A_{i}+B_{i} X_{a_{i}}{ }^{2}\right)^{\frac{1}{2}}\right]^{2} \frac{B_{i}}{\beta^{2}}-\frac{A_{i}}{B_{i}} \\
& V_{i}^{2}\left(X_{\mathrm{f}}\right)=\frac{1}{X_{\mathrm{f}}{ }^{2}}\left[\left(V_{i-1}{ }^{2}\left(X_{a_{i}}\right)-\gamma b_{i}\right) X_{a_{i}}{ }^{2}+\gamma b_{i} X_{\mathrm{f}}{ }^{2}\right]
\end{aligned}
$$

where

$$
i=1,2, \ldots, n-1 \quad \text { for } X_{a_{i}} \leq X_{\mathrm{f}}<X a_{i+1}
$$

and

$$
\begin{aligned}
& A_{i}=\left[V_{i-1}{ }^{2}\left(X_{a_{i}}\right)-\gamma b_{i}\right] X_{a_{i}}{ }^{2} \\
& B_{i}=\gamma b_{i} .
\end{aligned}
$$

After all of the pressure impulse has been applied to the snow, the particle velocity is

$$
V_{n}\left(X_{\mathrm{f}}\right)=\frac{\sum_{i=0}^{n-1} H_{p_{i}}}{\rho_{\mathrm{f}}(1-\beta) X_{\mathrm{f}}} \text { for } X_{a_{n}}<X_{\mathrm{f}}
$$

where

$$
\sum_{i=0}^{n-1} H_{p_{i}}=H_{p}
$$

is the total momentum per unit area applied to the snow. Equations (28) and (29) are used to start the solution and the recursion relations (Equations (30) and (31)) are used to follow the progression of the shock wave. Equation (32) is used to calculate particle velocities after the pressure impulse has ended.

Figure 4 (exponentials A and B) shows the results of using Equation (28) through (32), (2) and (7) to calculate the shock-wave attenuation for an exponentially decaying pressure impulse given by

$$
P=P_{0} \mathrm{e}^{-\alpha t}
$$

where $\alpha$ is a decay constant and $P_{0}$ is the initial pressure, and $f(t)=\mathrm{e}^{-\alpha t}$. Exponential $\mathrm{A}$ is the shock-wave pressure attenuation resulting from a single-stage compaction to $\rho_{\mathrm{f}}$ and exponential $\mathrm{B}$ is the attenuation that results from a multi-stage compaction. The exponential A and B shock waves each begin attenuating immediately upon application of the exponential pressure impulse. The attenuation for exponential A gradually increases to $X_{\mathrm{f}}^{-2}$ as the pressure impulse ends. Attenuation of exponential B is about $X_{\mathrm{f}}^{-1.5}$ after the pressure impulse ends, which is consistent with the results for instantaneous and finite-duration impulse and a multistage compaction. As with the constant-pressure impulse of finite duration, the attenuation of shock waves generated by finite-duration exponential pressure impulses depends on impulse duration. A short-duration impulse will produce a higher initial shock-wave pressure than an impulse with the same total momentum content but of longer duration.

\section{ATTENUATION OF CYLINDRICAL AND SPHERICAL GEOMETRY SHOCK WAVES}

Geometric spreading can greatly increase shock-wave attenuation in snow. Torvik (1971) developed equat- ions describing pressure attenuation for cylindrical and spherical geometry shock waves using a single-stage compaction snow-plow model and assuming instantaneous application of pressure impulse. For a cylindrically spreading shock, the pressure is given by

$$
\begin{gathered}
P(R)=\frac{I_{0}^{2} R_{0}^{2}(1-\beta)^{2}}{\rho_{0} \beta R^{2}(R-r)^{2}} \\
r=\left[\beta R^{2}+(1-\beta) R_{0}{ }^{2}\right]^{\frac{1}{2}} .
\end{gathered}
$$

The pressure for a spherically spreading shock wave is

$$
P(R)=\frac{I_{0}{ }^{2} R_{0}{ }^{4}(1-\beta)^{2}}{\rho_{0} \beta R^{4}(R-r)^{2}}
$$

where

$$
r=\left[\beta R^{3}+(1-\beta) R_{0}^{3}\right]^{\frac{1}{3}} .
$$

$I_{0}$ is the instantaneously applied pressure impulse, $R_{0}$ is the initial radius of the cavity surface in the snow on which the pressure impulse is applied, $R$ is the shockwave propagation radius and $r$ is the location of the inner radius of the cavity at some time after the application of the pressure impulse (Fig. 6).

When $R=R_{0}+\epsilon$, where $\epsilon \ll R_{0}$, a cylindrical geometry shock wave attenuates as $\epsilon^{-2}$ for single-stage compaction and about $\epsilon^{-1.5}$ for the multi-stage compaction curve shown in Figure 3, increasing to an attenuation of $R^{-4}$ for $\epsilon \gg R_{0}$ (Fig. 7). A spherical geometry shock wave attenuates as $\epsilon^{-2}$ for single-stage compaction and about $\epsilon^{-1.5}$ for multi-stage compaction when $\epsilon \ll R_{0}$, increasing to $R^{-6}$ for $\epsilon \gg R_{0}$ (Fig. 8). Pressure attenuation, for cylindrical and spherical shock waves, varies significantly depending on the initial radius over which the pressure impulse is applied and the distance from the initial radius.

\section{PRESSURE ATTENUATION OF A LINE CHARGE ON SNOW}

Line charges are used to clear minefields and to remove snow cornices from mountain ridge tops. Therefore, it is of practical interest to know the extent that snow atten-

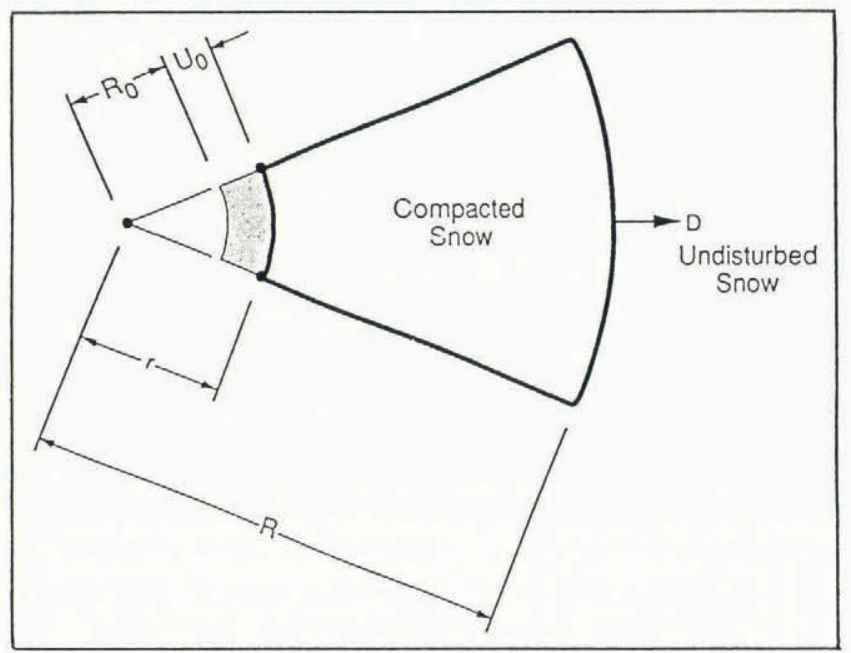

Fig. 6. Deformation geometry for diverging shock waves. 


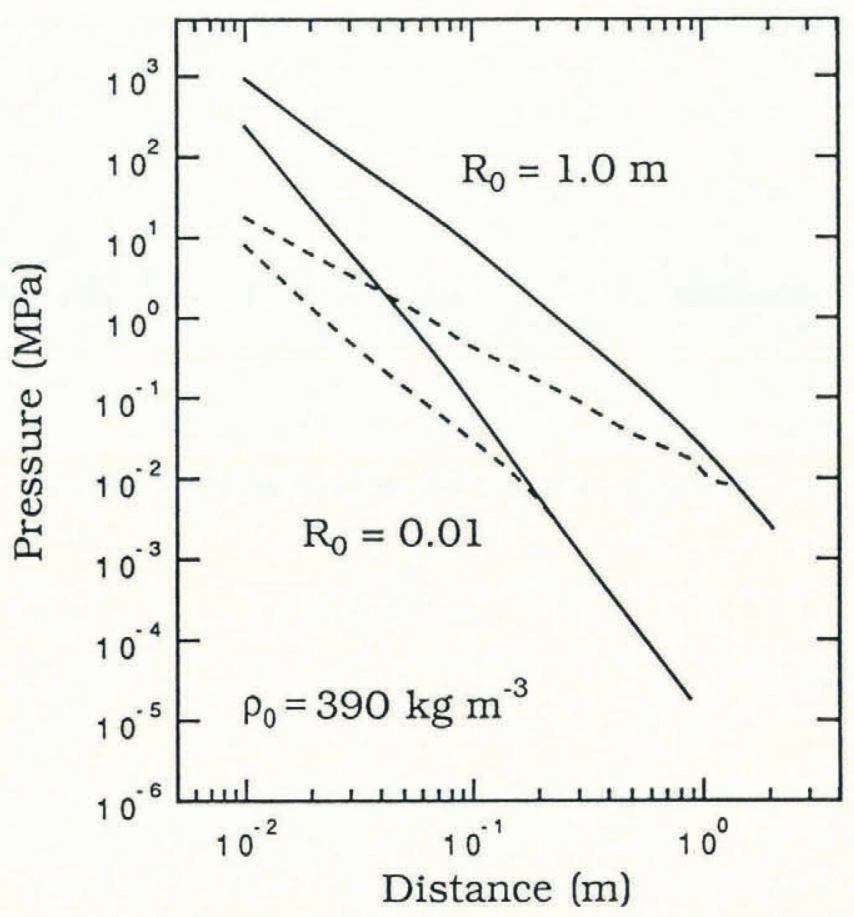

Fig. 7. Pressure attenuation with distance for instantaneously applied cylindrical pressure impulse (605 Pas) with initial radii of 0.01 and $1.0 \mathrm{~m}$, predicted by the snow-plow model. The solid lines represent the results for single-stage compaction and the dashed lines represent the results for multi-stage compaction.

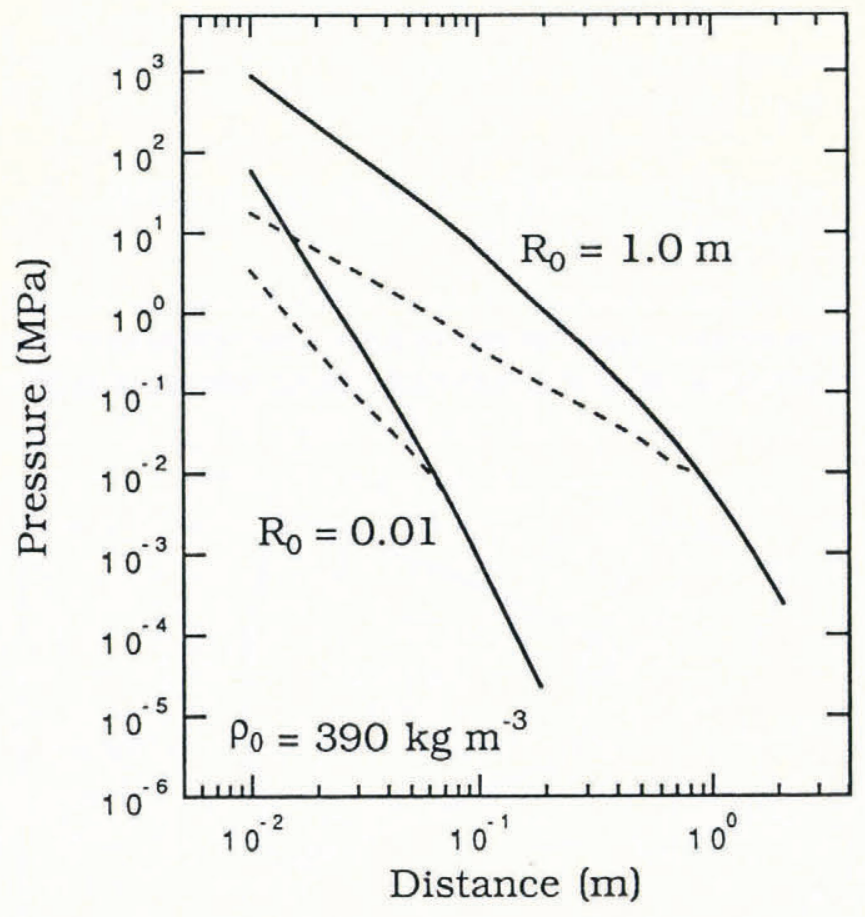

Fig. 8. Pressure attenuation with distance for instantaneously applied spherical pressure impulse (605 Pas) with initial radii of 0.01 and $1.0 \mathrm{~m}$, predicted by the snow-plow model. The solid lines represent the results for single-stage compaction and the dashed lines represent the results for multi-stage compaction.
Table 1. Air-blast measurements - shot 2 (after Ford, 1986)

\begin{tabular}{cccc}
$\begin{array}{c}\text { Distance } \\
\text { from } \\
\text { line charge }\end{array}$ & $\begin{array}{c}\text { Positive- } \\
\text { phase } \\
\text { duration }\end{array}$ & $\begin{array}{c}\text { Peak } \\
\text { air-blast } \\
\text { pressure }\end{array}$ & $\begin{array}{c}\text { Peak } \\
\text { impulse }\end{array}$ \\
$\mathrm{m}$ & $\mathrm{ms}$ & $\mathrm{MPa}$ & $\mathbf{k ~ P a ~ s}$ \\
\hline & & & \\
0.96 & 1.6 & 3.4 & 0.61 \\
2.99 & 11.0 & 1.64 & 1.36 \\
6.97 & 16.0 & 0.36 & 0.76 \\
11.00 & 18.0 & 0.16 & 0.61 \\
23.97 & 29.2 & 0.07 & 0.60 \\
\hline
\end{tabular}

uates shock waves produced by line-charge detonations. For a line charge resting on or above a snow cover, the cylindrical pressure wave that hits the snow surface propagates primarily through the air.

Ford (1986) has measured air-blast pressure, positivephase duration and total pressure impulse at the ground as a function of lateral distance from the axis of a linecharge explosion (Table 1). The positive-phase duration is defined as the duration of the compressive (positive) shock-wave pressure. It is assumed, for this example, that the air-blast pressure given in Table 1 would also occur over snow. Furthermore, the snow is assumed to be deep and shock-wave pressures are determined only for small propagation depths into the snow so that $\epsilon \ll R_{0}$, where $R_{0}$ is the distance from the line charge to the point of interest on the snow surface. With these conditions, the snow-ground boundary can be neglected and shock-wave pressure in the snow can be estimated from the equations for a one-dimensional variable-pressure impulse of finite duration (Equations (28)-(32), (2) and (7)), and an exponentially attenuating pressure pulse (Equation (33)). Figure 9 shows the shock-wave pressure (air blast) as a function of distance from the line charge $\left(d_{\mathrm{LC}}\right)$ along the snow surface, using the pressure-impulse data from Table 1. Figure 9 also shows the calculated pressures as a function of $\left(d_{\mathrm{LC}}\right)$ for shock-wave propagation depths into the snow of $0.06,0.2,0.4$ and $0.6 \mathrm{~m}$ calculated using the compaction curve given in Figure 3.

Air-blast shock-wave pressures do not attenuate the same amount for a given depth in the snow for different values of $\left(d_{\mathrm{LC}}\right)$. Shock-wave attenuation is greatest near the line charge $\left(d_{\mathrm{LC}}=0.96 \mathrm{~m}\right)$ and least at the farthest distance $\left(d_{\mathrm{LC}}=23.97 \mathrm{~m}\right)$. This occurs because the duration of the applied air-blast pressure impulse (positive-phase duration) increases with $d_{\text {LC }}$ (Table 1). The increasing positive-phase duration allows the shock wave to propagate through a greater depth of snow before starting the $\epsilon^{-1.5}$ attenuation. These findings suggest that, for shallow shock-wave penetration depth into a snow cover, line-charge pressure reduction depends on the positive-phase duration in addition to the total pressure impulse. Also, shock-wave pressure attenuation in a shallow snow cover can be reduced or eliminated by in- 


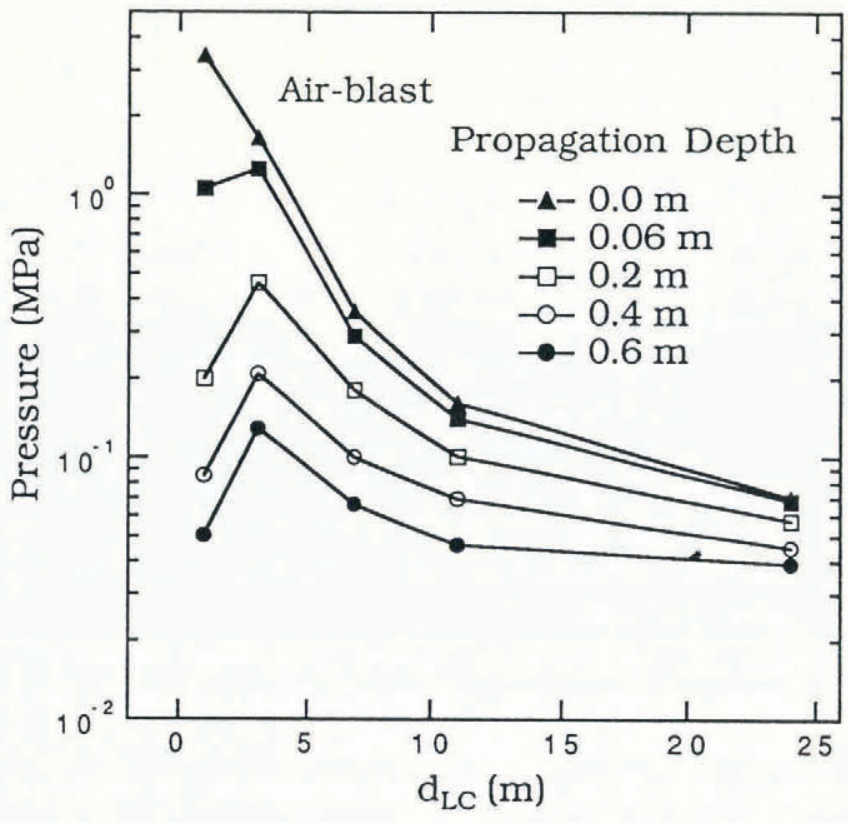

Fig. 9. Pressure attenuation with distance from the line charge for different shock propagation depths into the snow, predicted by the snow-plow model using exponential pressure impulses and multi-stage compaction. The air-blast curve is drawn from data given in Table 1.

creasing the positive-phase duration of a given pressure impulse.

\section{DISCUSSION AND CONCLUSIONS}

The snow-plow model has a limited ability to describe shock-wave propagation and attenuation in snow because of the simplifying underlying assumptions. The model is, however, capable of illustrating some of the important features and giving conservative estimates of shockwave attenuation in snow. Shock-wave pressure attenuation predicted by the snow-plow model will be less for a multi-stage snow compaction than for a single-stage compaction to the final snow density.

The snow-plow model predicts that maximum shockwave pressure attenuations will proceed as a function of $X_{\mathrm{f}}^{-2}$ for plane waves (where $X_{0}=0$ ), $R^{-4}$ for cylindrical waves, and $R^{-6}$ for spherical waves with a singlestage snow compaction. Attenuation for multi-stage compaction depends on the form of the $(\mathrm{P}-\rho)$ curve used to describe snow compaction and ranges from a function of $X_{\mathrm{f}}^{-1.5}$, for the compaction curve used in this study, to the maximum for plane, cyclindrical and spherical shock waves.

Comparison of instantancous, finite-duration squarewave and finite-duration exponential pressure impulses (each with the same total momentum) shows that shockwave attenuation can be markedly different during the period of pressure-impulse application. The instantaneously applied pressure impulse applies all of its momentum at once, resulting in infinite initial pressure that immediately begins attenuating as $X_{\mathrm{f}}^{-2}$ for single-stage compaction and as $X_{\mathrm{f}}^{-1.5}$ for multi-stage compaction.
The square-wave presure impulse maintains a constant pressure equal to the applied pressure until all of its momentum has been transferred to the snow and then begins to attenuate. Finally, the exponential pressure impulse gradually attenuates while its momentum is being transferred to the snow, asymptotically approaching an $X_{\mathrm{f}}^{-2}$ attenuation for single-stage compaction and an $X_{\mathrm{f}}^{-1.5}$ attenuation for multi-stage compaction. Once a pressure impulse has been applied to the snow, pressure attenuation is controled by the mechanical properties of the snow and the magnitude of total pressure impulse.

Mellor (1977) estimated that spherical geometry shocks, where $\epsilon \gg R_{0}$, attenuate as $R^{-3}$ to $R^{-4}$ as compared to the snow-plow model prediction of $R^{-6}$. It may be that the snow-plow model predictions are grossly in error because of the simple snow-compaction path used in the model or that the field measurements used by Mellor to make his estimates of attenuation were not the result of shock-wave propagation in snow. The pressure sensors used in many of the field tests could not survive near-source pressures and were, consequently, located well outside the zone of shock-wave-induced snow compaction. Outside this zone of snow compaction, pressure attenuation is due to viscous dissipation and geometric spreading that produce much less pressure attenuation than the pore-collapse mechanisms described by the snow-plow model. If, in fact, this was the case, Mellor's estimates of pressure attenuation in snow for spherical shocks may be too low, but a final conclusion is not possible until better snow-compaction and release paths are used in the model calculations, or more field tests are conducted.

Estimating shock-wave attenuation requires some thoughtful application of the results of this study. Explosive-induced shocks in snow often result from an explosive-charge detonation on the snow or in the air above the snow. In this situation the shock wave will be transmitted into the snow primarily by an air-blast wave propagating over the snow surface. If the initial radius for the air-blast pressure wave is large compared to the snow depth, then the shock wave may be approximated by a plane wave rather than a spherical wave or cylindrical wave. Attenuation will then proceed as $\epsilon^{-1.5}$ rather than as $R^{-6}$ for spherical waves or $R^{-4}$ for cylindrical waves.

Pressure attenutation of shock waves in snow can be reduced, delayed or even eliminated by using a sufficiently long-duration pressure impulse. Shock-wave propagation and attenuation in snow are affected by shock-wave geometry, pressure-impulse time variation and the mechanical properties of snow. Consequently, determining the mechanical response of snow to shock loading requires an accurate knowledge of the shockloading conditions in addition to a knowledge of stresses and particle velocities in the snow.

\section{ACKNOWLEDGEMENTS}

I thank Dr J.A. Brown (Los Alamos National Laboratory), Dr E.S. Gaffney (Ktech Corporation) and K. Jones (CRREL) for technically reviewing this paper. The research described in this paper was funded by DA Project 4A762784AT42, "Cold regions engineering tech- 
nology"; Work unit CS/012, "Attenuation of shock waves by snow".

\section{REFERENCES}

Brown, J.A., E.S. Gaffney, G.L. Blaisdell and J.B. Johnson. 1987. Techniques for gas gun studies of shock wave attenuation in snow. Proceedings of the American Physical Society Topical Conference on Shock Compression of Condensed Matter, Monterey, California, July 20-24, 1987, 657-660.

Brown, R.L. 1980. An analysis of non-steady plastic shock waves in snow. J. Glaciol., 25(92), 279-287.

Brown, R. L. 1981. A method for evaluating shockwave propagation in snow. Cold Reg. Sci. Technol., 5(2), 151-156.

Brown, R. L. 1983. A comparison of unsteady wave propagation for various snowpack properties. Ann. Glaciol., 4, 30-36.

Ford, M.B. 1986. Mine-clearing performance of XM268 blasting agent line charge. Vicksburg, Mississippi, USA Waterways Experiment Station. (TR SL-86-4.)

Hanagud, S. 1967. Snow as a locking material: high pressure properties of snow. In Ōura, H., ed. Physics of snow and ice. International Conference on Low Temperature Science ... August, 14-19, 1966, Sapporo, Japan. Proceedings. Vol. I. Part 2. Sap- poro, Hokkaido University, Institute of Low Temperature Science, 807-826.

Herrmann, W. 1971. Constitutive equations for compaction of porous materials. Albuquerque, New Mexico, Sandia National Laboratory. (Report SC-DC71 4134.)

Hornbeck, P. W. 1971. Numerical methods. New York, Quantum Publishers.

Johnson, J. B., J. A. Brown and E. S. Gaffney. 1990. Interpretation of the stress histories from shock impact tests on snow using embedded stress gauges. Proceedings of the American Physical Society Topical Conference on Shock Compression of Condensed Matter, Albuquerque, New Mexico, Aug. 14-17, 1989, 117-120.

Kolsky, H. 1963. Stress waves in solids. New York, Dover Publications. (Republication of work from Clarendon Press, Oxford, 1953.)

Mellor, M. 1977. Engineering properties of snow. J. Glaciol., 19(81), 15-66.

Torvik, P.J. 1971. On the attenuation of diverging shock waves. Wright-Patterson Air Force Base, Ohio, U.S. Air Force Institute of Technology. (Report AFIT TR 71-4.)

The accuracy of references in the text and in this list is the responsibility of the author, to whom queries should be addressed.

MS received 19 March 1990 and in revised form 19 September 1990 